\title{
PLURALISMO, MULTICULTURALISMO \\ E ESTRANEI
}

\author{
di Giovanni Sartori
}

\section{Pluralismo e tolleranza}

Il pluralismo è di moda. Ma le parole troppo popolari diventano oggetto di contesa e di conquista - dopotutto nomina numina - e così vengono abusate, distorte e trivializzate. Pertanto è bene ricostruire il concetto di pluralismo nella sua genesi e nel suo sviluppo storico, e così ancorare la parola alla storia della parola. Dal che risulterà che per quanto il concetto sia sempre stato, per così dire, elastico, non ne consegue che «pluralismo» sia una parola tuttofare atta a nobilitare quasiasi causa. A tirarli troppo anche gli elastici si rompono. Analogamente, più di tanto il concetto di pluralismo non può essere stiracchiato. $\mathrm{O}$ così sembra a me.

Storicamente, l'idea di pluralismo (sottolineo: l'idea, non la parola, che arriverà secoli più tardi) è già implicita nello sviluppo del concetto di tolleranza e nella sua graduale accettazione nel XVII secolo nel solco delle guerre di religione ${ }^{1}$. Si capisce che tolleranza e pluralismo sono concetti diversi; ma è anche facile capire che sono intrinsecamente connessi. In questo senso: che il pluralismo presuppone tolleranza, e quindi che un pluralismo intollerante è un falso pluralismo. La differenza è questa: che la tolleranza rispetta i valori altrui, mentre il pluralismo afferma un valore proprio. Perché il pluralismo afferma che la di-

Ripubblichiamo con lievi ritocchi e ampliamenti il testo di una lettura alla Accademia dei Lincei tenuta a Roma il 7 marzo 1997, che compare negli Atti dell'Accademia con il titolo «Capire il Pluralismo».

1 I classici lavori sulla tolleranza sono l'Areopagitica di Milton (1644), l'Epistola de Tolerantia di Locke (1689), e il Traité sur la Tolerance di Voltaire (1763). Tre recenti volumi collettanei tra loro connessi sono: Horton e Mendus (1985); Edwards e Mendus (1987); e Mendus (1988).

RIVISTA ITALIANA DI SCIENZA POLITICA / a. XXVII, n. 3, dicembre 1997 
versità e il dissenso sono valori che arricchiscono l'individuo e anche la sua città politica.

Si deve sottolineare che questo è un radicale rovesciamento di prospettive. E molti attribuiscono il merito di questa inversione di prospettive alla Riforma e specificamente al puritanesimo. Il più eminente sostenitore di questa tesi è stato A.D. Lindsay $(1934)^{2}$. Ma si deve stare attenti nel generalizzare. La Riforma protestante pluralizza le chiese; e in questa rottura e frammentazione non c'è nulla di intrinsecamente pluralistico. Quanto al puritanesimo, se si fa specifico riferimento alla esperienza delle congregazioni e delle comunità puritane, allora deve essere chiaro che per la maggior parte dei puritani inglesi e americani «democrazia» e «libertà» erano parole e ideali spregevoli. $\mathrm{E}$ vero che i puritani affermavano la libertà di coscienza e di opinione; ma in realtà rivendicavano la libertà della propria coscienza e opinione, per essere poi intolleranti nei confronti delle opinioni e religioni altrui. E dunque non c'è nulla di necessariamente pluralistico nello sfidare le autorità costituite in nome della libertà di coscienza. Perché quel che rivendichiamo per noi stessi può essere negato ad altri.

L'esperienza puritana è stata importante, invece, nel rompere il nodo tra la sfera di Dio e quella di Cesare, e in questo senso nel depoliticizzare la società. Con i puritani il centro di gravità della vita umana si colloca in associazioni volontarie indipendenti dallo Stato; associazioni il cui legame interno (tra associati) prevale sul legame (esterno) tra individui e Sovrano. $\mathrm{Ma}$ questa depoliticizzazione non implica - lo ripeto - che i puritani abbiano scoperto la visione pluralistica del mondo. D'altra parte, scoprire i padri fondatori non interessa più di tanto. Interessa invece capire bene il significato e la straordinaria novità della scoperta.

Fino al XVII secolo si era sempre ritenuto che la diversità fosse causa della discordia e dei disordini che portavano gli Stati alla rovina. Pertanto si era sempre ritenuto che la salute dello Stato postulasse l'unanimità. Ma in quel secolo si andò gradualmente affermando una concezione opposta e fu l'unanimità che

2 Tale interpretazione è stata di recente ripresa da Maddox (1996). La tesi che fa risalire l'origine della democrazia al puritanesimo si fonda soprattutto sull'esperienza dei Levellers e sui Putney Debates (accolti, insieme ad altri libelli dei puritani di sinistra, in Gabrieli 1956). Questa letteratura dell'età di Cromwell è certo caratterizzata da forti elementi libertari; ma non fa testo per l'insieme dell'esperienza puritana. 
divenne man mano sospetta. E la civiltà liberale, e poi la liberal-democrazia, sono state costruite a pezzi e bocconi sulla base di questo rivoluzionario giro di boa. Gli imperi dell'antichità, le autocrazie, i despotismi, sono portatori di (e sorretti da) una visione monocromatica della realtà, mentre la democrazia è multicolore. Ma è la democrazia liberale, non la democrazia degli antichi, che si fonda sul dissenso e sulla diversità. Siamo noi, non i Greci dell'età di Pericle, ad avere inventato un sistema politico di concordia discors, di consenso alimentato da dissenso.

\section{Il pluralismo partitico}

Fin qui, a volo d'uccello, una storia di idee. Ma come è che queste idee si sono tradotte in fatti, in realtà? Una risposta è di guardare alla nascita dei sistemi partitici, a come e perché i partiti sono diventati partiti.

I partiti si dicono tali perché «parti». E quando sosteniamo che il dissenso e la diversità sono «buone» per il corpo sociale e per la città politica, il sottinteso è che la città politica è fatta, e anzi è bene che sia fatta, di parti. E quelle parti che chiamiamo partiti si sono affermate, storicamente, in forza di quel sottinteso.

Beninteso, tutti gli ordinamenti politici hanno sempre dispiegato al loro interno gruppi in lotta spietata tra loro. Ma questi gruppi erano chiamati, in politica, fazioni. Come è, allora, che le fazioni si trasformano in partiti? Il nome cambia perché la cosa cambia. Peraltro, sia il nome che la cosa si sono affermati molto lentamente. Il termine partito appare all'inizio del XVIII secolo, e viene in evidenza con la Dissertation upon Parties di Bolingbroke del 1733-34; ma è solo con Burke nel 1770 (in Thoughts on the Cause of Present Discontents) che i partiti vengono dichiarati per la prima volta non solo necessari ma anche «rispettabili». Nella sua celebre definizione Burke dice così: «Partito è un corpo di persone unite per promuovere, con il loro comune impegno, gli interessi nazionali in base a uno specifico principio sul quale tutti convengono». Così Burke distingue nettamente il partito dalla fazione. Le fazioni rappresentano soltanto «una lotta meschina e interessata per la conquista di posti e di rimunerazioni», mentre i partiti sono honorable connections, onorevoli connessioni «necessarie per il pieno disbrigo del nostro dovere pubblico» (1839, vol. 1, 425-426).

Quando Burke, così scrivendo, contravveniva alla comune 
opinione del suo tempo che i partiti degenerano sempre in fazione (e che sono come fazioni) asserendo invece che ne erano il superamento, questa intuizione non aveva un sostegno dottrinario, un retroterra teoretico. Siamo noi, retrospettivamente, che afferriamo come il passaggio dalla fazione al partito presuppone l'affermarsi di una Weltanshauung pluralistica. Fuori dal pluralismo il partire, il dividersi e parteggiare, è cattivo, è essere parte contro l'intero, a danno dell'intero, e cioè fazione. È soltanto con il pluralismo che diventa concepibile il dividersi «buono», e così i partiti come parti $d i$ un intero, come componenti positive del loro intero. I partiti sono inconcepibili nella città di Hobbes e non venivano contemplati nella città di Rousseau. I partiti vengono in essere solo quando si afferma la credenza che un mondo variegato e molteplice è migliore di un mondo monocromatico. Dunque pluralismo e partiti sono, idealmente, nati da un parto. E dire «pluralismo partitico» è espressione pregnante. Sta per dire che i partiti al plurale sono un prodotto «reale» del pluralismo come ideale ${ }^{3}$.

\section{L'impoverimento del concetto}

Torniamo alla Begriffsbildung, alla costruzione concettuale. Storicamente, si è visto, il concetto di pluralismo si sviluppa lungo la traiettoria che va dalla intolleranza alla tolleranza, dalla tolleranza al rispetto del dissenso e poi, tramite quel rispetto, al credere nel valore della diversità. Ma quando la parola pluralismo venne coniata e poi incorporata, nel nostro secolo, nel vocabolario della politica, gli antenati intellettuali che ho menzionato furono ignorati o dimenticati. I primi pluralisti inglesi degli anni '20 (Figgis, D.H. Cole e soprattutto Harold Laski) derivarono la loro dottrina dal Genossenschaftsrecht tedesco teorizzato da Gierke, e cioè dal mondo medievale delle corporazioni, e pertanto ridussero il pluralismo a una teoria della società multi-gruppo intesa a negare il primato dello Stato. Questa riduzione è, per la Begriffsbildung, accettabile; ma certo costituisce un drastico impoverimento del concetto. E i successivi pluralisti americani degli anni '50 (ben rappresentati dal volume The Governmental Process di David Truman) fecero peggio. Nella ver-

3 Utilizzo qui Sartori (1976, 3-13). 
sione politologica americana (sorvolo su quella degli antropologi, che aggiungerebbe solo confusione a confusione), il pluralismo comincia con Arthur Bentley (che scriveva The Process of Government nel 1908) e approda a una pura e semplice teoria dei gruppi di interesse, alla cosiddetta interest group theory of politics ${ }^{4}$. E qui usciamo davvero dal seminato. A parte il fatto che far partire il pluralismo da Bentley è storiograficamente risibile, se pluralismo è espressione e rivendicazione di «interesse» allora tutta la nobiltà del concetto è perduta. In realtà, nel cosiddetto pluralismo americano non c'è nessun contenuto distintivamente pluralistico. Del pluralismo come credenza di valore non c'è più alcuna traccia, il concetto diventa completamente disancorato dalla sua ragion d'essere, e così una parola librata al vento che suona bene ma che significa poco.

Il che concorre a spiegare la grande popolarità acquisita dalla parola dagli anni '60 in poi. Da allora ci viene raccontato che il pluralismo c'è sempre e ovunque. C'è in Africa ${ }^{5}$, c'è in India, c'era nella Unione Sovietica (a dispetto del comunismo) ${ }^{6}$, e c'è dappertutto per forza (e cioè per definizione) perché tutte le società sono in qualche modo «plurali»e in qualche modo differenziate.

Sì, ma soprattutto e fondamentalmente no. Pluralismo non è essere plurali. E se confondiamo i due concetti allora mettiamo assieme, in una notte hegeliana nella quale tutte le vacche sono nere, una frammentazione tribale (Africa), un sistema di caste (India) e anche (perché no?) l'esistenza conforme al proprio stato dell'ordine medievale. Ma questa è soltanto una operazione che io chiamo di vaporizzazione dei concetti, e cioè di distruzione delle idee chiare e distinte. Io non ci sto. E prima di riprendere il cammino e di arrivare ai più recenti abusi del termine, $\mathrm{mi}$ incombe di precisare che cosa si possa e debba sensatamente intendere per «pluralismo».

4 Vedi in breve, Gunnell (1996). Il punto non è sulla qualità della letteratura in questione, ma su quanto poco contenga di propriamente pluralistico.

5 Vedi, per tutti, Kuper e Smith (1969).

6 Per uno sguardo d'insieme, cfr. Solomon (1983). Ho criticato gli studi sovietici, in questo e altri rispetti, in Sartori (1993). 


\section{Livelli di analisi}

Premetto e ripeto che derivare «pluralismo» da «plurale» da qualsiasi più-che-uno - è soltanto espressione di povertà culturale. E per capire il pluralismo estraendolo dal grande magma «tutto-pluralistico» che ho richiamato sopra, andrò a distinguere tra tre livelli di analisi, e cioè tra $i$ ) pluralismo con credenza, ii) pluralismo sociale, e iii) pluralismo politico.

Al livello dei sistemi di credenza si può parlare di una cultura pluralistica con la stessa latitudine di significato con la quale discorriamo di una cultura secolarizzata. Difatti le due nozioni sono complementari. Se una cultura è secolarizzata, non può essere monistica. Viceversa, se è pluralistica deve essere secolarizzata (le fedi rivelate non tollerano contro-fedi). Comunque sia, a livello di credenze questa latitudine di significato si precisa così: che una cultura pluralistica è tanto più genuina quanto più si richiama al suo retroterra storico, e cioè al principio della tolleranza. Che la varietà e non l'uniformità, il dissentire e non l'unanimità, il variare e non l'immobilismo, siano «cose buone», queste sono le credenze di valore che emergono con la tolleranza, che si ascrivono al contesto culturale del pluralismo, e che devono essere espresse da una cultura pluralistica che non usurpa il nome che si dà. E queste sono le premesse in base alle quali dobbiamo valutare il cosiddetto «multiculturalismo» dei nostri giorni ${ }^{7}$.

In teoria, o in linea di principio, è chiaro che il pluralismo dovrebbe sottoscrivere una molteplicità culturale. Ma nella misura in cui l'odierno multiculturalismo è aggressivo, separante e intollerante, nella stessa misura il multiculturalismo in questione è la negazione stessa del pluralismo. Il pluralismo sostiene e alimenta una società aperta (diciamo popperiana) che riflette un «ordine spontaneo» (nel senso teorizzato da Hayek), e certamente rispetta una società multiculturale che c'è. Tuttavia l'intento primario del pluralismo è di assicurare la pace inter-culturale, non di fomentare una ostilità tra culture. I liberals americani che difendono il multiculturalismo (tra i quali il più eminente è Charles Taylor) parlano di una «politica del riconoscimento» (recognition) ${ }^{8}$. Ma convenientemente dimenticano di precisare che un contesto pluralistico postula un riconoscimen(1996).

7 Una antologia interdisciplinare sul tema è quella a cura di Gordon e Newfield

8 Vedine il contributo in Gutman (1994). 
to reciproco. Un riconoscimento che viene ricambiato da un radicale disconoscimento è anti-pluralistico. L'attacco a testa bassa contro gli autori «maschi, bianchi e morti» che sono stati gli autori canonici della civiltà occidentale (ivi includendo Dante e Shakespeare, per citare solo due nomi) è soltanto espressione di radicale incultura; e redimerlo sotto il manto del pluralismo è analfabetismo o altrimenti disonestà intellettuale. Ripeto: il pluralismo è figlio della tolleranza, e quindi è «vocato» a disconoscere una intolleranza che è poi, in effetti, un odio culturale che rivendica una superiorità culturale alternativa.

Naturalmente i multiculturalisti ci raccontano che il loro è un «neo-pluralismo». E la novità sarebbe che l'antefatto è diverso. Sheldon Wohlin osserva che la tolleranza lockiana si ascrive a una pluralità di associazioni volontarie, e cioè a «identità che non ci obbligano», mentre il nuovo pluralismo si riferisce ad associazioni involontarie (di sesso o di razza) che ci restano «appiccicate addosso» $(1993,467)$. Vero, ma fino a un certo punto. Le associazioni del tempo di Locke (e fino al 1789) non erano per nulla volontarie e si iscrivevano ad una società rigidamente stratificata di ceti e di corporazioni (dalla quale non si usciva più facilmente di quanto non si possa fare oggi dal sesso - operandosi - o dal colore della pelle). Comunque sia, il punto è che qualsiasi «identità» (volontaria o involontaria) è trattata dal pluralismo allo stesso modo e cioè, dicevo, in termini di rispetto e di riconoscimento reciproco. Se così non è, allora pluralismo non è. Pertanto si deve ripetere che un multiculturalismo che rivendica la secessione culturale, e che si risolve in una tribalizzazione della cultura, è anti-pluralistico. Il cosiddetto neo-pluralismo non può in alcun modo redimere anche se si applica a circostanze diverse - la negazione del pluralismo. E chiamarlo neo-pluralismo è imbrogliare.

Passo al secondo livello di analisi e cioè al pluralismo sociale. Il punto è, qui, che non dobbiamo confondere il pluralismo sociale con una qualsiasi differenziazione sociale. Siccome non esistono società di eguali (salvo che negli scritti utopici), tutte le società sono variamente differenziate. Non ne consegue che siano tutte differenziate «pluralisticamente». Sul punto tornerò. Al momento osservo soltanto che è sbagliato ritenere che tutte le società siano, in qualche misura, inevitabilmente pluralistiche. Per carità! Il pluralismo non è un puro e semplice equivalente della nozione di «complessità strutturale». È, invece (vedremo), un tipo specifico di struttura sociale. 
E vengo al terzo livello di analisi, al pluralismo politico. In prima approssimazione possiamo dire che a livello politico il termine pluralismo denota una diversificazione del potere (nella terminologia di Robert Dahl una «poliarchia aperta») che si fonda su una pluralità di gruppi che sono, ad un tempo, indipendenti e non-esclusivi. Ho già indicato come questo pluralismo politico riplasma le «parti» in partiti. E quindi passo ad altri punti specifici.

Un primo punto verte su come il pluralismo si riflette sul consenso e sul conflitto. Si è spesso sostenuto che la democrazia si fonda sul conflitto, non sul consenso. Non concordo, e qui vedo un uso mistificante, o quanto meno troppo diluito, della nozione di conflitto. Il conflitto, quello vero, induceva Hobbes ad accettare una pace imposta dal dominio dispotico del suo Leviatano; e il conflitto induceva Bolingbroke e Hume, Madison e Washington (e su su fino a Benedetto Croce), a diffidare del «partizzare» e ad invocare una «coalizione dei partiti». Quando conflitto è conflitto, e cioè un quissimile di guerra, allora non aiuta per nulla a costruire la città liberal-democratica. Dunque deve essere chiaro che l'elemento centrale della Weltanschauung pluralistica non è né il consenso né il conflitto ma, invece, la dialettica del dissentire, e per essa un dibattere che in parte presuppone consenso e in parte assume intensità di conflitto, ma che non si risolve in nessuno di questi due termini.

In verità, consenso e conflitto acquistano una funzione e una rilevanza diversa a livelli diversi di analisi. A livello di fundamentals, di principii fondamentali, occorre il consenso. E il consenso più importante di tutti è il consenso sulle regole di risoluzione dei conflitti (che è, in democrazia, la regola maggioritaria). Dopodiché, se c'è consenso su come risolvere i conflitti, allora è lecito «confliggere» sulle policies, sulla soluzione delle questioni concrete, a livello di politiche di governo. Ma è così perché il consenso di fondo, o sui fondamenti, ci autolimita nel confliggere, e cioè addomestica il conflitto, lo trasforma in conflitto pacifico. Per converso, e d'altro canto, il consenso non deve essere inteso come un parente prossimo della unanimità. Il consenso pluralistico si fonda su un processo di aggiustamenti tra menti e interessi dissenzienti. Potremmo dire così: consenso è un processo di sempre mutevoli compromessi e convergenze tra persuasioni divergenti.

Un secondo punto verte sul rapporto tra pluralismo e regola maggioritaria, che in inglese (majority rule) si precisa come 
una regola-comando. Se il comando maggioritario è inteso come lo fu da Madison, Tocqueville, e da John Stuart Mill, e cioè come la minaccia di una tirannide della maggioranza, di una concreta maggioranza numerica che «comanda» nel senso letterale del termine, allora il pluralismo rifiuta la tirannide della maggioranza. Il che non implica che il pluralismo respinga il principio (si badi, il principio) maggioritario come principio regolativo, vale a dire come criterio decisionale 9 . Ovviamente no. Anche così il pluralismo si pone come la migliore difesa e legittimazione del principio maggioritario limitato, del principio che la maggioranza deve rispettare i diritti della minoranza, e quindi del principio che la maggioranza deve esercitare il suo potere con moderazione nei limiti posti dal rispetto del principio pluralistico.

Un terzo punto verte sul nesso tra pluralismo e la «politica come pace» (e non come guerra, come nella versione hobbesiana e schmittiana della politica). Il pluralismo, si è detto all'inizio, divide la sfera di Dio da quella di Cesare, e così facendo nega che il Vescovo o il Principe abbiano una «pretesa totale» su di noi. Con il passar del tempo questa negazione o limitazione viene sempre più a tutelare una sfera privata dell'esistenza, tale che le alterne vicende della lotta politica non mettono più a repentaglio i beni e la vita stessa dei contendenti. Insomma, chi perde può tornare tranquillamente a casa. Ed è a quel momento che viene in essere una politica di pacifica rotazione e sostituzione al potere, e per essa la città pluralistica. Lo ridico così: la città pluralistica presuppone che le varie sfere della vita - i domini della religione, della politica e della economia - siano adeguatamente separati; e questi sono presupposti che sono stati affermati dal pluralismo (anche se, va da sé, non soltanto dal pluralismo).

Un ultimo punto, il quarto, investe la già accennata configurazione strutturale del pluralismo. Una società frammentata non è per questo una società pluralistica. E se è vero, come è vero, che il pluralismo postula una società di «associazioni multiple», questa non è una determinazione sufficiente. Infätti queste associazioni debbono essere, in primo luogo, volontarie (non obbligatorie o di chi ci nasce dentro) e, in secondo luogo, non-esclusive, aperte a affiliazioni multiple. E quest'ultimo è il tratto di-

9 Vedi, più ampiamente, Sartori (1987, cap. VI, specialmente 131-137). 
stintivo. Dunque una società multi-gruppo è pluralistica se, e soltanto se, i gruppi in questione non sono gruppi tradizionali, e, secondo, solo se si sviluppano «naturalmente» senza essere in alcun modo imposti. Dal che si ricava che il cosiddetto pluralismo africano non è tale, e che nemmeno lo è un sistema di stratificazione di caste (leggi: India).

Il punto può essere condensato in questo indicatore: l'esistenza o meno di cross-cutting cleavages, e cioè di linee di divisione intersecanti (o incrociate). Difatti l'assenza di cleavages intersecanti è un criterio che consente da solo di escludere dal pluralismo tutte le società la cui articolazione si fonda su tribù, razza, casta, religione e qualsiasi tipo di gruppo tradizionalistico. Il che non è detto per discriminare contro chicchessia, ma perché il pluralismo funziona solo se c'è, e non funziona se inventato e mal attribuito. Dunque, il pluralismo funziona quando i cleavages, le linee di divisione, sono neutralizzate e frenate $\mathrm{da}$ affiliazioni (e anche lealtà) multiple, mentre disfunziona, per così dire, quando le linee di frattura economico-sociali coincidono, sommandosi e rinforzandosi l'una con l'altra (per esempio in gruppi la cui identità è congiuntamente etnica, religiosa, linguistica, e/o di ricchi contro poveri). In questo caso la pace e la coesistenza sociale può ancora essere assicurata da élites consociative (era il caso, per esempio, dell'Olanda). Ma la pace sociale è in pericolo quando le «comunità chiuse» poste da cleavages coincidenti diventano invasive e aggressive.

Tutto ciò precisato, si deve sempre tener presente che $\mathrm{i}$ cross-cutting cleavages indicano un elemento strutturale, non uno stato di credenze; e che la credenza nel valore del pluralismo è la condizione pregiudiziale di tutto il resto.

\section{Tolleranza, consenso e comunità}

Capire il pluralismo è anche capire, allora, di tolleranza, consenso, dissenso e conflitto. Vorrei ora brevemente approfondire i primi due concetti, e poi introdurre nel discorso la nozione di comunità.

Ridiamo, per cominciare, un'occhiata alla tolleranza. Tolleranza non è indifferenza, né presuppone indifferenza. Se siamo indifferenti non siamo interessati: fine del discorso. Nemmeno è vero, come spesso si sostiene, che la tolleranza presuppone un relativismo. Certo, se siamo relativisti siamo aperti a una molte- 
plicità di punti di vista. Ma la tolleranza è tolleranza (lo indica il nome) proprio perché non presuppone una visione relativistica. Chi tollera ha credenze e principii propri, li ritiene veri, e tuttavia concede che altri hanno il diritto di coltivare «credenze sbagliate». Il punto è importante perché stabilisce che il tollerare non è, né può essere, illimitato. «La tolleranza è sempre in tensione e non è mai totale. Se una persona tiene a qualcosa, cercherà di farla accadere; altrimenti è difficile ritenere che davvero ci tenga. Ma non cercherà di farla accadere con qualsiasi mezzo, ad ogni costo» (Lucas 1985, 296-301).

Allora, qual è l'elasticità della tolleranza? Se la domanda ci fa cercare un confine fisso e prestabilito, quel confine non lo troveremo. Il grado di elasticità della tolleranza può essere stabilito, invece, da tre criteri. Il primo è che dobbiamo sempre fornire ragioni di quel che consideriamo intollerabile (e cioè la tolleranza vieta il dogmatismo $)^{10}$. Il secondo criterio coinvolge il harm principle, il principio «di non far male», di non danneggiare. Insomma, non siamo tenuti a tollerare comportamenti che ci infliggono danno o torto. E il terzo criterio è sicuramente la reciprocità: nell'essere tolleranti verso altri ci aspettiamo, a nostra volta, di esserne tollerati.

Passiamo ora a ridare un'occhiata al consenso. L'inglese ci permette di distinguere tra consensus e consent, diciamo tra uno stato diffuso di consenso, e un puntuale e concreto consentire. Distinzione che ci aiuta a precisare che il consenso in questione non è un attivo approvare e sostenere questo o quello. Pertanto il consenso può essere pura e semplice accettazione, un concorrere generalizzato e soltanto passivo. Anche così, il consenso è un condividere che in qualche modo lega (Graham 1984). E questa definizione mette bene a fuoco il collegamento tra il concetto di consenso e quello di comunità.

Si noti: anche la comunità può essere definita «un condividere che in qualche modo lega». E il mio discorso deve approdare, per essere completo, alla nozione di comunità perché non possiamo più assumere che l'unità politica per eccellenza sia lo Stato-nazione. Il che ci costringe a ripensare il problema. E, per

10 Su basi parallele John Rawls distingue fra «pluralismo ragionevole» e «pluralismo come tale» e sostiene il primo perché una società liberal-democratica si basa su una serie di «punti di vista» universali che richiedono la lealtà di tutti (1993, 36-39). Sono d'accordo sulla sostanza, ma secondo me è il pluralismo come tale che è (e deve essere) «ragionevole». 
ripensarlo, occorre far capo a quella unità primaria di tutte le costruzioni politiche che è, appunto, la comunità.

Per quanto lo Stato-nazione sia o ci sembri tuttora importante, il fatto è, in prospettiva, che lo Stato-nazione si è costituito solo nel corso del XIX secolo, e che la felix Austria, l'impero polietnico e multinazionale degli Asburgo, ha retto benissimo (quantomeno combattendo bene) fino alla sconfitta del 1919. Dunque, lo Stato-nazione è stato il principio organizzativo unificante dello Stato moderno - soltanto o soprattutto in Europa - per meno di due secoli. Prima, e a partire dal Medioevo, nationes erano le lingue. La nazione tedesca era coloro che parlavano in tedesco, e così per tutti. Lo Stato-nazione fu concepito dal romanticismo - ché l'Illuminismo fu cosmopolita - e si concepisce come una entità che non è soltanto linguistica. Nella sua versione diciamo finita, lo Stato-nazione è una entità organica (evocata dalle nozioni di «spirito del popolo», di Volksgeist e di Volkseele), radicata in un mitico, lontano passato, e rinforzata (dalla Rivoluzione francese) dalla passione patriottica, e ancor più rinforzata - nella sua versione estrema - da una «identità di sangue» (razziale, e quindi da non confondere con l'innocuo principio giuridico del jus sanguinis).

$\mathrm{Su}$ queste premesse «nazione» si trasforma in «nazionalismo» e - nel suo sviluppo in Germania con Hitler - in purezza e supremazia razziale. Ma quella di Hitler è stata una estremizzazione solitaria. Il grosso degli Stati nazionali emersi in Europa sulla scia delle Rivoluzioni del 1830 e del 1848, affermano soltanto una identità linguistica e patriottica. Nazione è stata, per i più, una rivendicazione di indipendenza che ha distrutto gli aggregati puramente dinastici che si erano andati costituendo nell'età dell'assolutismo. Con lo Stato-nazione non è più concepibile che i popoli «passino di mano» non solo su basi di conquista (così può ancora accadere), ma di successione e eredità dinastica. Per ottenere un paese, nel XVII secolo, bastava un matrimonio. Così, fortunatamente, non è più. $\mathrm{Ma}$ i meriti passati dello Stato-nazione non bastano oggi a salvarlo come «unità ottimale» della geopolitica. Perché sta di fatto che lo Stato-nazione è assoggettato, oggi, a un duplice svuotamento: verso il più piccolo e anche verso il più grande.

Sia come sia, il mio punto è soltanto questo: che tanto più la «comunità nazionale» si indebolisce, e tanto più dobbiamo cercare o ritrovare una comunità. Ridetto per esteso: ogni volta che una sovrastruttura (la nazione, l'impero o altro) si disgrega, 
noi torniamo inevitabilmente alla infrastruttura primordiale che i greci chiamavano koinonia e risorge il bisogno di ritrovare una Gemeinschaft, un collante che «sentiamo» e che - dicevo - ci collega e ci lega.

Gemeinschaft era il concetto che Tönnies contrapponeva a Gesellschaft (società). La prima era, per lui, un «organismo vivente», mentre la società era soltanto un aggregato meccanico non più fondato su un immediato idem sentire ma su mediazioni di scambio e di contratto. Tönnies resta, sul concetto di comunità, il classico di riferimento. Ma la sua Gemeinschaft era soltanto, o comunque soprattutto, il «gruppo primario». Non nego che il significato forte del concetto si dispieghi nei gruppi simbiotici. Ma di «comunità» si dà anche un significato più debole che si estende al contesto che Ch.H. Cooley chiamava «gruppo secondario». Riformulo così: che la comunità di Tönnies è la comunità concreta, al di là della quale si dà anche la comunità astratta.

Pertanto, riprendendo il mio filo, non sto dicendo che dobbiamo tornare al piccolo, e che «il piccolo è bello». E vero che le comunità del passato (la polis greca, i comuni medievali, la democrazia di villaggio) erano micro-collettività che operavano faccia a faccia. Ma se la comunità non è concepita come un corpo operativo, ma come un identity marker, diciamo come un «identificatore», un comune sentire nel quale ci identifichiamo e che ci identifica, allora non occorre che una comunità sia piccola. Così Italiani, Inglesi, Francesi, Tedeschi, e via dicendo, possono essere concepiti come «larghe comunità» alla stessa stregua in cui sono o erano percepiti come nazioni; e per quanto la Comunità europea, o il parlare di una comunità latinoamericana, ci rinvii a «comunità astratte», se questi grandi aggregati sono partecipati e ci danno un senso di partecipazione, è lecitissimo considerarli come comunità, sia pure sui generis.

Sto dicendo, allora, che gli esseri umani vivono infelicemente nello stato di folle solitarie, in condizioni anomiche, e pertanto che cercano sempre di appartenere, di accomunarsi e di identificarsi in organizzazioni e organismi nei quali si riconoscono: per cominciare, in comunità concrete di vicinato, ma poi anche in estese «comunità simboliche». Senonché anche a questo effetto si pone un problema di elasticità analogo a quello nel quale ci siamo imbattuti in sede di tolleranza. In quella sede ci eravamo chiesti: qual è il limite oltre il quale la corda della tolleranza si spezza? Ora ci dobbiamo chiedere: fino a che punto possiamo tirare la corda della comunità? 
Così come non credo alla contrapposizione schmittiana tra Freund e Feind, tra amico e nemico ${ }^{11}$, nemmeno riesco a credere, all'altro estremo, alla diffusa apertura cosmopolitica auspicata dall'ultimo Dahrendorf. Parlare di comunità mondiale è pura retorica, è vaporizzare il concetto di comunità. A me pare, invece, che l'animale umano si aggrega in coalescenze e «sta assieme» sub specie di animale sociale, a patto che esista sempre un confine (mobile ma non cancellabile) tra noi e loro. Noi è la nostra identità; loro sono le identità dissimili che determinano la nostra. Siamo chi siamo in funzione di chi non siamo. Ogni comunità implica clausura, un raccogliersi assieme che è anche un chiudere fuori, un escludere. Un «noi» che non è circoscritto da un loro, nemmeno si costituisce.

\section{Comunità pluralistica e reciprocità}

Sono così pronto per la domanda più spinosa di tutte, vale a dire: in quale misura il pluralismo slarga e diversifica la nozione di comunità? Insomma, pluralismo e comunità come stanno tra loro? Una comunità può sopravvivere se spezzata in sotto-comunità che sono poi, in concreto, contro-comunità che arrivano a rifiutare le regole che istituiscono un convivere comunitario?

Nell'affrontare questo delicato quesito debbo ricordare che la comunità pluralistica è una acquisizione recente, difficile e ovviamente fragile $^{12}$. Una comunità pluralistica è definita dal pluralismo. E il pluralismo così come l'ho definito presuppone - lo ricordo - una disposizione tollerante e, strutturalmente, associazioni volontarie «non imposte», affiliazioni multiple, e cleavages, linee di divisione, trasversali e incrociate. Le comunità del passato - dalla polis greca alle comunità puritane - non avevano queste caratteristiche, anzi. Aggiungi che queste caratteristiche si dispiegano, a tutt'oggi, soltanto nel mondo occidentale o occidentalizzato. Che è proprio il mondo più esposto, oggi, a massicce immigrazioni dall'Est, ma soprattutto dall'Africa e dal Terzo mondo.

11 Cfr. la mia critica a Schmitt in Sartori (1995, 276-284).

12 Tanto recente che Tönnies, che scriveva nel 1887 (vedine la traduzione italiana Tönnies 1963), non l'aveva né intravista né contemplata. A Tönnies, così come per Durkheim e ancora, negli anni '20, per Max Weber, la nozione stessa di «comunità pluralistica» sarebbe sembrata un controsenso. 
Il caso, si dirà, non è senza precedenti; e dicendo così noi guardiamo soprattutto agli Stati Uniti. Sì, il nuovo mondo è tutto un mondo di «nuovi venuti»; e l'afflusso di immigranti negli Stati Uniti è stato davvero, in determinati periodi, massiccio. Nel periodo $1845-1925$ - in ottanta anni - circa 50 milioni di persone hanno traversato l'Atlantico; e negli anni 1900-1913 gli immigrati sono stati addirittura 10 milioni. Ma questi nuovi arrivati trovavano, nel nuovo mondo, uno sterminato spazio vuoto, cercavano e desideravano una nuova patria, ed erano felici di diventare americani: il melting pot, il calderone di fusione, con i 50 milioni di immigrati di cui ho detto, ha funzionato benissimo $^{13}$. Invece il vecchio mondo è da gran tempo un mondo senza spazi vuoti e un mondo di relativamente pochi «nuovi venuti». E quindi il precedente americano non ci aiuta ad affrontare il problema. Gli europei (dell'Ovest) sono preoccupati, si sentono invasi e stanno diventando reattivi.

Razzismo? È l'accusa sbrigativa di chi tiene sempre ad essere «politicamente corretto». Ma l'accusa è superficiale, generalizza troppo, e rischia di essere controproducente. Chi viene denunziato come razzista senza esserlo si infuria, e magari finisce per diventarlo davvero. Non dobbiamo generalizzare, ma invece precisare. Lo spettro delle reazioni ai nuovi venuti è vario e complesso. Per alcuni - molti - la reazione è difesa del posto di lavoro e del salario. Per altri è xeno-paura, un sentirsi insicuri e potenzialmente minacciati. Per altri ancora si tratta di una reazione di rigetto (xenofobia). Ed è solo da quel punto in là che ci imbattiamo in un razzismo. E anche quando il fenomeno è davvero di xenofobia e/o di razzismo, queste reazioni sono raramente a tutto campo. Gli immigrati dall'Est sono «resistiti» sul terreno economico, non razziale. La xenofobia si concentra invece sugli immigranti africani e islamici. Nel primo caso è spesso razziale (non piace una razza nera); ma nel secondo è soprattutto culturale. E questo è il vero nodo della questione. Finché non si arriva all'ultimo caso, la controversia è eminentemente di gradualità: quanti immigrati possono essere assorbiti in quanto tempo. Ma nel caso dei gruppi mobilitati o mobilita-

13 È oggi che non funziona più, e proprio in funzione di un multiculturalismo che rischia di frantumare la nazione americana. Come scrive Arthur Schlesinger «l'America si vede sempre di più come composta di gruppi che sono più o meno radicati nel loro carattere etnico» $(1992,16)$. 
bili dal fondamentalismo islamico la questione è un'altra. E va posta brutalmente.

La domanda è: fino a che punto una tolleranza pluralistica si deve piegare non solo a «stranieri culturali» ma anche ad aperti e aggressivi «nemici culturali»? Insomma, può il pluralismo accettare, arrivare fino ad accettare, la propria frantumazione, la rottura della comunità pluralistica? E una domanda simile a quella che nella teoria della democrazia si formula così: una democrazia deve consentire la propria distruzione democratica? E cioè deve consentire che $\mathrm{i}$ suoi cittadini votino per un dittatore?

Che una diversità sempre maggiore, e quindi radicale e radicalizzante, sia per definizione un «arricchimento» è una formula di sconvolgente superficialità. La mia tesi è, invece, che esiste un punto oltre il quale il pluralismo non può e non deve andare; e che il criterio, nella difficile navigazione che sono andato descrivendo, è essenzialmente quello della reciprocita ${ }^{14}$. Pluralismo è sì vivere assieme in differenza e con differenze; ma lo è - insisto - in contraccambio, rispettandosi. Entrare in una comunità pluralistica è, congiuntamente, un acquisire e un concedere. Stranieri che non sono disposti a concedere in cambio di quel che ottengono, che si propongono di restare «estranei» alla comunità nella quale entrano sino al punto di contestarne, quantomeno in parte, gli stessi principii, sono stranieri che inevitabilmente suscitano reazioni di rigetto, di paura e di ostilità. Il detto inglese è che il pasto gratis non esiste. Deve e può esistere una cittadinanza gratuita, concessa in cambio di nulla? A mio avviso, no.

\section{Riferimenti bibliografici}

Burke, E. (1839), The Works of Edmund Burke, Boston, Little Brown. Edwards, D. e S. Mendus (a cura di) (1987), On Toleration, Oxford, Clarendon Press.

Gabrieli, V. (a cura di) (1956), Puritanesimo e Libertà, Torino, Einaudi. Gordon, A.F. e C. Newfield (a cura di) (1996), Mapping Multiculturalism, Minneapolis, University of Minnesota Press.

14 Osserva esattamente Gian Enrico Rusconi: «Essere cittadini non significa soltanto fruire di beni-diritti soggettivi ma impegnarsi a contribuire alla loro produzione... è un comportamento intrinseco allo status di cittadini che riconoscono di avere vincoli di reciprocità» $(1996,21)$. 
Graham, G. (1984), Consensus, in G. Sartori (a cura di), Social Science Concepts, Beverly Hills, Sage.

Gunnell, J. (1996), The Genealogy of American Pluralism: From Madison to Behavioralism, in «International Political Science Review», n. 3.

Gutman, A. (a cura di) (1994), Multiculturalism: Examining the Politics of Recognition, Princeton, Princeton University Press.

Horton, J. e S. Mendus (a cura di) (1985), Aspects of Toleration, London, Methuen.

Kuper, L. e M.G. Smith (a cura di) (1969), Pluralism in Africa, Berkeley, University of California Press.

Lindsay, A.D. (1934), The Churches and Democracy, London, Epworth Press.

Lucas, J.R. (1985), The Principles of Politics, Oxford, Clarendon Press.

Maddox, G. (1996), Religion and the Rise of Democracy, London, Routledge.

Mendus, S. (a cura di) (1988), Justifying Toleration, Cambridge, Cambridge University Press.

Rawls, J. (1993), Political Liberalism, New York, Columbia University Press.

Rusconi, G. (1996), Multiculturalismo e cittadinanza democratica, in «Teoria Politica», n. 3.

Sartori, G. (1976), Parties and Party Systems, New York, Cambridge University Press.

- (1987) The Theory of Democracy Revisited, Chatham, Chatham House.

- (1993), Totalitarianism, Model Mania and Learning from Error, in «Journal of Theoretical Politics», n. 1.

- (1995), Elementi di Teoria Politica, Bologna, Il Mulino ( $3^{\text {a }}$ ed.).

Schlesinger, A. (1992), The Disuniting of America: Reflections on a Multicultural Society, New York, Norton.

Solomon, S. (a cura di) (1983), Pluralism in the Soviet Union, New York, St. Martin's Press.

Tönnies, A. (1963), Comunità e Società, Milano, Comunità.

Wohlin, S. (1993), Democracy, Difference, and Re-Cognition, in «Political Theory», n. 3. 